\title{
Realization of Via-free Microstrip Composite Right/Left-Handed Transmission Lines
}

\author{
Mark A. Eberspächer, Kim Eccleston*, Thomas F. Eibert \\ Lehrstuhl für Hochfrequenztechnik, Technische Universität München \\ Arcisstr. 21, 80333 München, Germany \\ mark.eberspaecher@tum.de \\ *Department of Electrical and Computer Engineering, University of Canterbury, Christchurch, New Zealand
}

\begin{abstract}
Three via-free microstrip composite right/left handed (CRLH) unit cells are presented, which enable easy and low-cost fabrication of transmission lines exhibiting the infinite wave length phenomenon. All guiding structures are designed in an entirely printed circuit technology based on the transmission line theory and its equivalent circuit. Supported by EM simulation software the unit cells are optimized to operate at a frequency of $10 \mathrm{GHz}$. Appropriate experiment boards were fabricated and the measurements obtained from a microwave vector network analyzer are compared to the simulated results. Furthermore, an approach is worked out to determine the dispersion characteristics of the unit cells without the need of an equivalent circuit. The introduced and applied method allows to calculate dispersion relations directly based on simulated and measured ABCD parameters. Left and right-handed regions are pointed out by the dispersion diagram and the so-called infinite wavelength phenomenon is exhibited.
\end{abstract}

\section{INTRODUCTION}

In general, transmission lines with some predefined behavior can be realized by cascading appropriate LCnetworks periodically. Theoretically, the properties of these arrangements are determined by the elements of the LCnetworks which are often referred to as unit cells. In order to gain an artificial TL, which appears as a homogenous structure the physical length $p$ of the unit cells should be less than a quarter of the guided wavelength [1].

Interchanging the series inductance and the shunt capacitance in the equivalent circuit model of a conventional TL with short length leads to its dual known as a left-handed (LH) transmission line. However, a practical realization of such a unit cell with a purely LH behavior is impossible since parasitic parallel capacitance and serial inductance always appear. Thus the realizable artifact always consists of an LH and a conventional RH contribution. Consequently it is named as CRLH unit cell, which is depicted in Fig. 1.

A link between the elements of the unit cell and the propagation constant of the realized transmission line can be derived with the approach for periodic structures according to [2]. In general, $L_{L} C_{R} \neq L_{R} C_{L}$ hold and this results in the propagation constant

$\gamma=\alpha+\mathrm{j} \beta=\frac{\sqrt{Z Y}}{p}=\frac{\mathrm{j}}{p} \sqrt{\left(\omega L_{R}-\frac{1}{\omega C_{L}}\right)\left(\omega C_{R}-\frac{1}{\omega L_{L}}\right)}$
It can be shown that this expression can be simplified for $L_{L} C_{R}=L_{R} C_{L}$, which is called the balanced case. On that condition the propagation constant reads

$\gamma=j \beta=\frac{\omega}{p} \sqrt{L_{R} C_{R}}-\frac{1}{p} \frac{1}{\omega \sqrt{C_{L} L_{L}}}=\beta_{R}+\beta_{L}$.

Since for $\omega=\frac{1}{\sqrt{L_{R} C_{L}}}=\frac{1}{\sqrt{L_{L} C_{R}}}$ the propagation constant

becomes zero the relationship $\lambda_{g}=2 \pi /|\beta|$ corresponds with an infinite guided wavelength, which is the so-called infinite wavelength phenomenon.

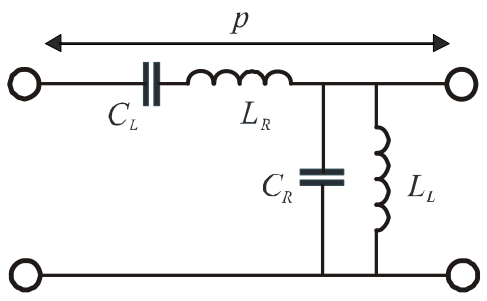

Fig. I Equivalent circuit of a composite right/left-handed unit cell with the physical length $p$.

As seen above, the propagation constant can be determined easily when the values of the equivalent circuit of the structure are known. However, in many cases these parameters are not available or it is difficult to determine them. This is mainly a problem if the structure is completely realized without any discrete elements, for example in microstrip technique.

Unlike the equivalent circuit, the ABCD-parameters of arbitrary unit cell can be extracted easily by EM Simulation or measurement. Hence it is convenient to base further calculation on these parameters. This provides also the big advantage that the whole structure, including host lines and even distributed configuration, can be taken into account. For reciprocal networks, which have the physical length $p$ and are arranged periodically, the relationship

$$
\cosh [(\alpha+j \beta) p]=\frac{A+D}{2}
$$

is valid according to [2]. In general, the parameters $A$ and $D$ are complex and the problem can be solved by determining 
the principal value of the complex inverse cosh-function. The imaginary part of this result corresponds to $\beta$.

\section{REALIZATION OF VIA-FREE CRLH UNIT CELLS}

The basic idea, which have all three guiding structures in common, is to realize the shunt inductance by open-ended stubs and using them simultaneously to perform the required series capacitance. Thus, interlayer connections become superfluous and via-free designs are possible. In the following three different approaches are introduced. All unit cells are designed to be produced on Taconic TLY-5 substrate with $\mathrm{DK}=2.2$ and $\mathrm{DF}=0.0009$.

\section{A. Structure I}

As seen in Fig. 2, the first proposed CRLH unit cell is realized by installing the inductive stubs alternately from both sides of the host microstrip lines, so that capacitive coupling occurs.

In general the odd-mode equivalent circuit of two coupled microstrip lines comprises two different capacitances. One capacitance is located between the microstrip lines and the ground conductor and a second one between the coupled microstrip lines. Compared with the equivalent circuit in Fig. 1 and applying this analysis to the proposed unit cell the first named capacitance contributes to $C_{R}$, while the last one mainly determines $C_{L}$. Additionally, $C_{R}$ comprises the host line's capacitance contributions and parasitic capacitance. Thus, $C_{R}$ increases easily and the goal is given by generating a sufficient series capacitance $C_{L}$. According to [3], a small ratio of transmission line width to substrate height $(w / h)$ reduces $C_{R}$ without influencing $C_{L}$. Whereas the distance between two fingers and the substrate height form the ratio $s / h$ which should be small in order to reach a sizeable value of $C_{L}$. The height $h$ is given by the used substrate with $1.57 \mathrm{~mm}$, while the minimum dimension of $w$ and $s$ are restricted to $0.3 \mathrm{~mm}$ by the available fabrication process.

So far, only the width and the clearance of the finger lines are discussed. However, for designing the inductance their lengths $l$ referred to the guided wavelength $\lambda_{g}=20.2 \mathrm{~mm}$ (10 GHz). Clearly, the lengths have to be chosen in the range between $\frac{\lambda_{g}}{4}<l<\frac{\lambda_{g}}{2}$, i.e. $5.05 \mathrm{~mm}<l<10.1 \mathrm{~mm}$, to achieve an inductive behavior. The exact number of stubs and their lengths are optimized within the mentioned restrictions by EM simulation.

The dispersion diagrams of the structure, seen in Fig. 3, are computed based on the simulated and measured ABCD parameters. The corresponding S-parameters of the simulated and measured unit cell are depicted in Fig. 4. It is seen in the dispersion diagram that the infinite wave length phenomenon occurs approximately $400 \mathrm{MHz}$ below the predicted frequency at $9.6 \mathrm{GHz}$. This can be explained by further analysis of the produced unit cell which shows that the structure doesn't possess the simulated dimension exactly.

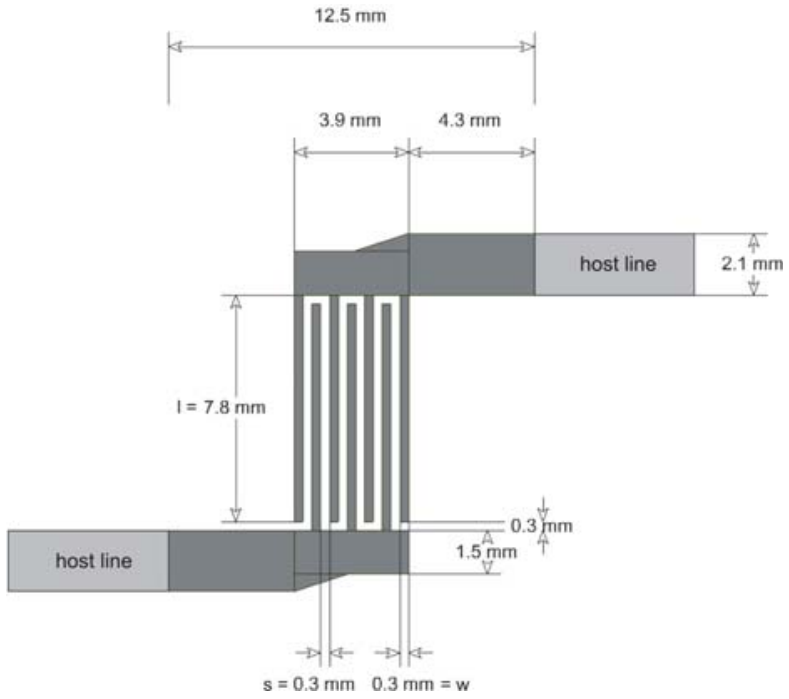

Fig. 2 Layout of the via-free microstrip CRLH unit cell Structure I (substrate thickness $h=1.57 \mathrm{~mm}$ )

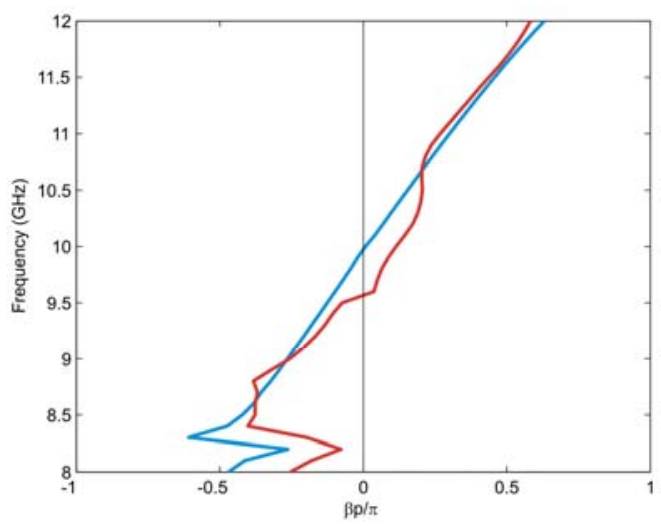

Fig. 3 Dispersion diagram of the CRLH unit cell Structure I, (blue) based on EM simulation, (red) based on measurements.

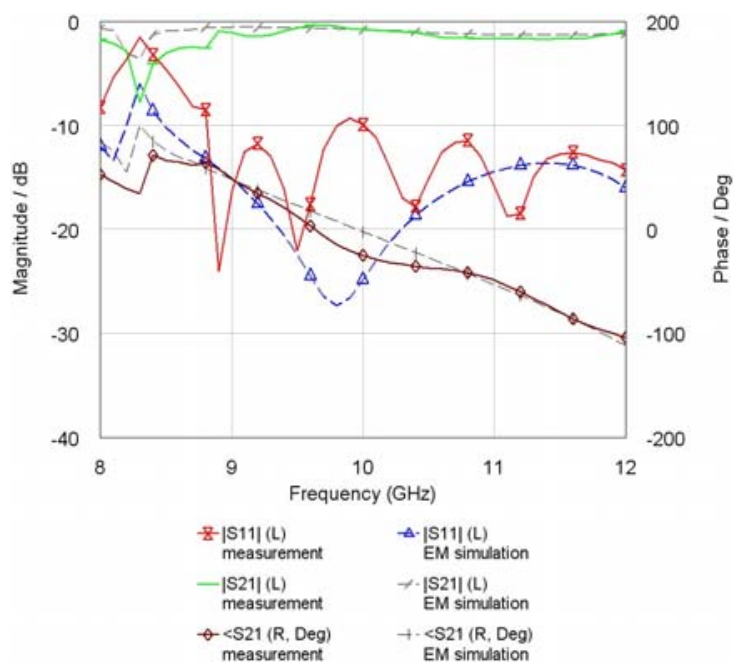

Fig. 4 Simulation and experimental results of the via-free CRHL unit cell Structure I (reference impedance $=83 \Omega$ ). 


\section{B. Structure II}

In contrast to the first design this structure II, seen in Fig. 5, is realized with only two open-ended stubs per unit cell. The capacitive coupling of the two stubs is achieved by a pattern known as an interdigital capacitor. Since the capacitor should be seen as a lumped element for an incident wave, its finger length must be smaller than $\lambda_{g} / 8$ according to [3]. Due to [4] the maximum of capacitance density can be achieved if the finger width $w$ and the space $s$ between the fingers are chosen equally. In addition, the width should be selected as small as possible to reduce the arising parasitic shunt capacitance. The lower limit of the available fabrication process is given as $0.3 \mathrm{~mm}$, which dictates this dimension. Realizing the inductance by open-ended stubs can be done in a similar way to the procedure of structure I. To decrease occurring radiation loss of this wave guiding structure, the substrate height is reduced to $0.78 \mathrm{~mm}$. A big advantage of this design is the possibility to optimize capacitance and inductance more independently by manipulating the stub lengths or the interdigital fingers.

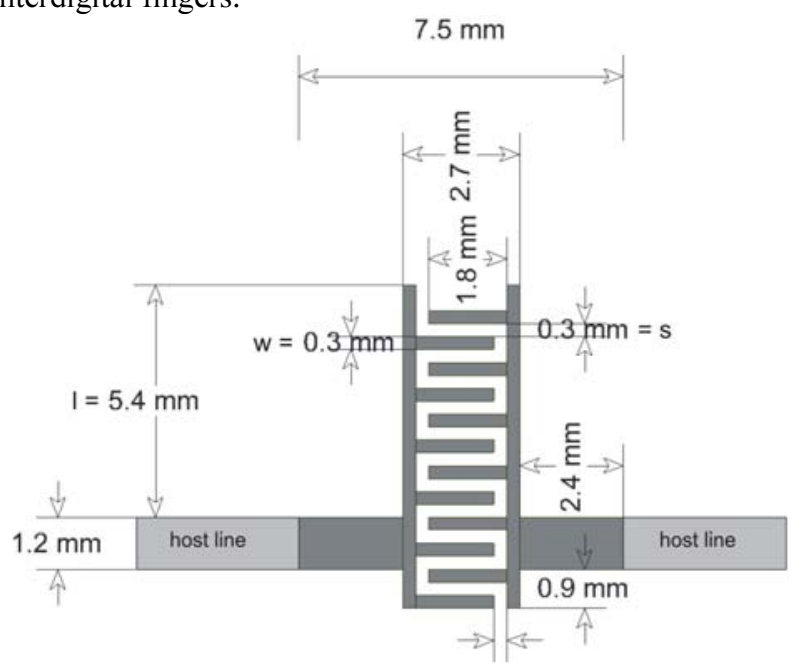

$0.3 \mathrm{~mm}$

Fig. 5 Layout of the via-free microstrip CRLH unit cell Structure II (substrate thickness $h=0.78 \mathrm{~mm}$ )

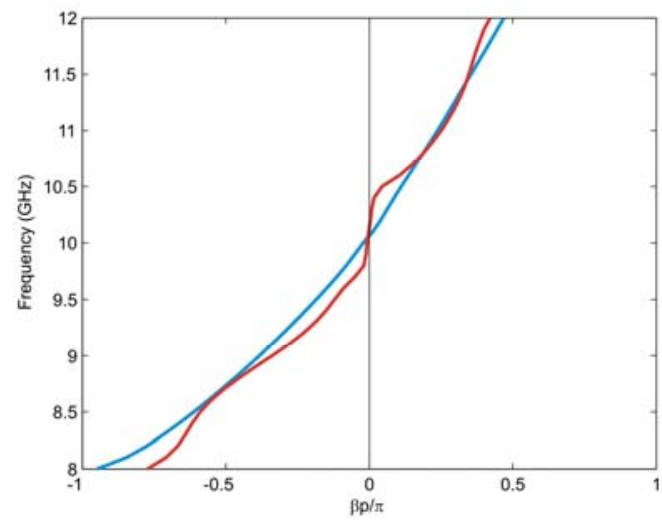

Fig. 6 Dispersion diagram of the CRLH unit cell Structure II, (blue) based on EM simulation, (red) based on measurements.

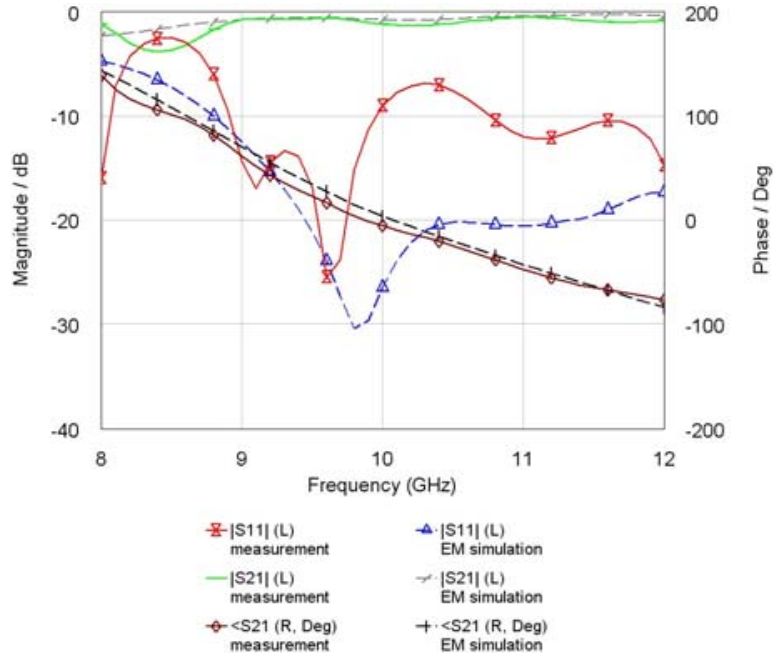

Fig. 7 Simulation and experimental results of the via-free CRHL unit cell Structure II (reference impedance $=60 \Omega$ )

The dispersion diagrams and the S-parameters are shown in Fig. 6 and Fig. 7, respectively. They are depicted both, as measured and simulated data. The observable step in the measured dispersion diagram points out that the produced unit cell does not exactly fulfill the conditions for the balanced case. The explanation for this is given by a detailed view of the produced structure which shows that the fabrication process was not sufficient accurate.

\section{Structure III}

The layout of the proposed third unit cell is displayed in Fig 8. The structure consists of two isolated conducting stripes which are arranged in parallel. Hence a series capacitance is exhibited, in which the magnitude is determined mainly by the clearance of the conductors and their common length. Considering the structure with the transmission line theory in mind it represents two host lines each loaded with two openended stubs. Depending on the length $l_{i}(i \in\{1,2\})$ of these stubs either an additional capacitive shunt or an inductive shunt behavior occurs. For $l_{i}<\frac{\lambda_{g}}{4}$ a capacitance is realized, for $\frac{\lambda_{g}}{4}<l_{i}<\frac{\lambda_{g}}{2}$ an inductance. As depicted in Fig 8, each side of the feed transmission line contains one capacitive stub and one inductive stub. While the inductance is desired, the arising parallel capacitance is parasitic. However it cannot be erased with this approach. Indeed it is possible to take this capacitance into account with the capacitance $C_{R}$, but an oversized enlargement of this parameter will lead to an unbalanced unit cell. Hence a reduction in width of the transmission line, seen in Fig. 8, is introduced to exhibit a series inductance, which balances the influence of the increased parallel capacitance. The dimension of the step in width and the stub length are optimized by using EM simulation software. 


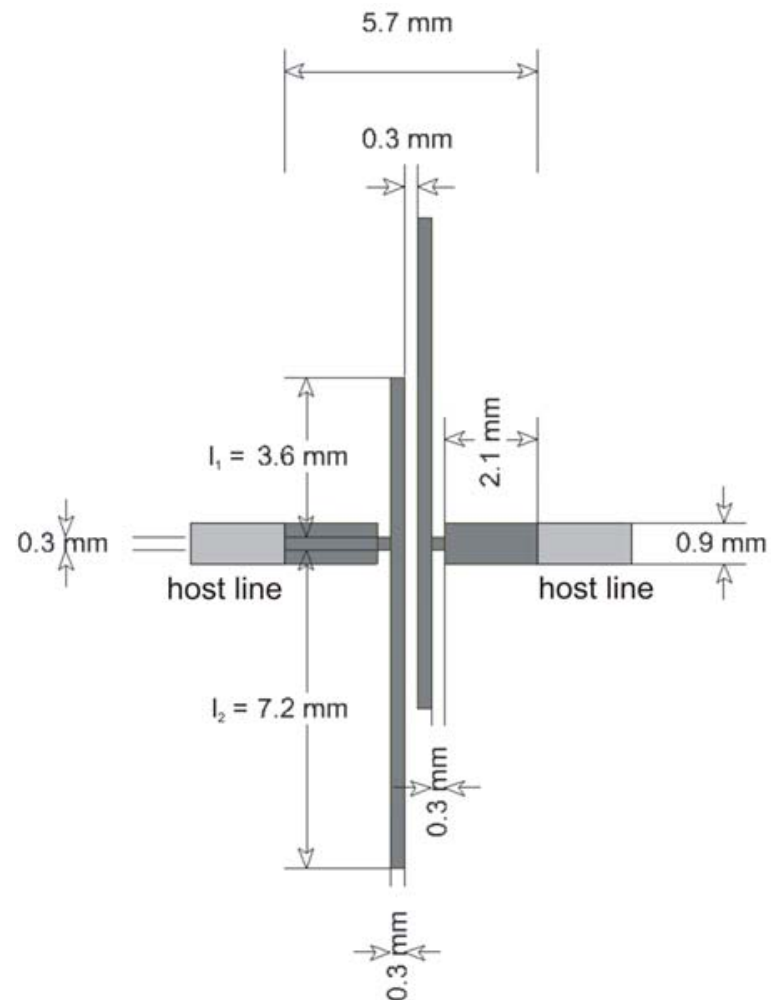

Fig. 8 Layout of the via-free microstrip CRLH unit cell Structure III (substrate thickness $h=0.78 \mathrm{~mm}$ ).

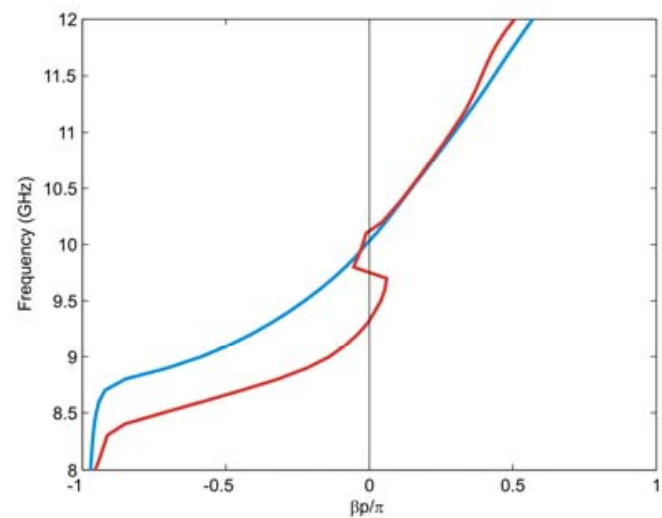

Fig. 9 Dispersion diagram of the CRLH unit cell Structure III, (blue) based on EM simulation, (red) based on measurements.

It is seen in Fig. 9 that the infinite wave length phenomenon occurs as predicted by the simulation at a frequency of $10 \mathrm{GHz}$. However, the measured phase differs strongly from the simulation around $9 \mathrm{GHz}$. This can be considered in the resulting S-parameters in Fig. 10 as well as in the dispersion diagram. Additionally, a comparison of both diagrams shows that the determination of the dispersion diagram is influenced by computation errors in the region of the axis $\beta p / \pi=0$.

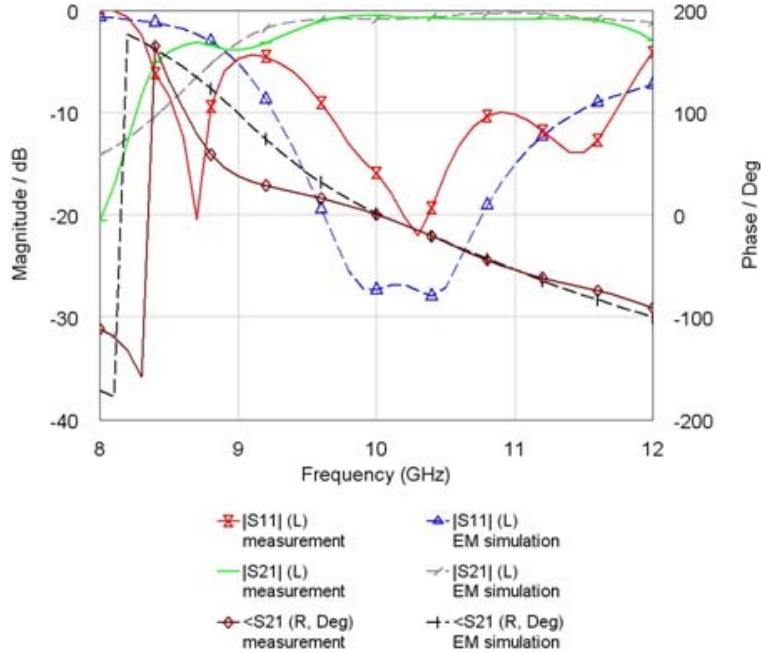

Fig. 10 Simulation and experimental results of the via-free CRHL unit cell Structure III (reference impedance $=90 \Omega$ ).

\section{CONCLUSIONS}

In this work, three one-dimensional CRLH unit cells operating at $10 \mathrm{GHz}$ were developed. All three presented guiding structures are entirely based on microstrip technology and can be implemented without an interlayer connection. Consequently, an extra fabrication process is superfluous and costs can be saved. The performance of the infinite wave length phenomenon have been predicted by full-wave simulation and proven experimentally. It is found that slight differences between both results are caused by inaccuracies of the fabrication process.

\section{REFERENCES}

[1] A. Lai and T. Itoh, "Composite Right/Left-Handed Transmission Line Metamaterials," IEEE Microwave Mag., pp. 34-50, Sep. 2004.

[2] David M. Pozar, Microwave Engineering ( $3^{\text {rd }}$ ed.) New York: Wiley, 2005

[3] J-S. Hong, M.J. Lancaster, Microstrip filters for RF/Microwave applications, New York: Wiley, 2001, pp85,86, 97

[4] G.D. Alley, "Interdigital Capacitors and Their Application to LumpedElement Microwave Integrated Circuits", IEEE Trans. Microwave Theory and Techniques, vol. 18, pp1028-1033, December 1970 